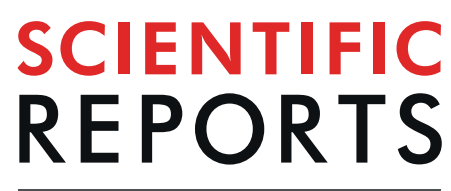

natureresearch

\title{
OPEN The Simon Effect Based on Allocentric and Egocentric Reference Frame: Common and Specific Neural Correlates
}

Received: 11 October 2018

Accepted: 4 September 2019

Published online: 24 September 2019

\author{
Hui Li ${ }^{1}$, Nan Liu ${ }^{1}$, You Li ${ }^{1}$, Ralph Weidner ${ }^{2}{ }^{2}$, Gereon R. Fink ${ }^{2,3}$ \& Qi Chen ${ }^{1,4}$
}

An object's location can be represented either relative to an observer's body effectors (egocentric reference frame) or relative to another external object (allocentric reference frame). In non-spatial tasks, an object's task-irrelevant egocentric position conflicts with the side of a task-relevant manual response, which defines the classical Simon effect. Growing evidence suggests that the Simon effect occurs not only based on conflicting positions within the egocentric but also within the allocentric reference frame. Although neural mechanisms underlying the egocentric Simon effect have been extensively researched, neural mechanisms underlying the allocentric Simon effect and their potential interaction with those underlying its egocentric variant remain to be explored. In this fMRI study, spatial congruency between the task-irrelevant egocentric and allocentric target positions and the task-relevant response hand was orthogonally manipulated. Behaviorally, a significant Simon effect was observed for both reference frames. Neurally, three sub-regions in the frontoparietal network were involved in different aspects of the Simon effect, depending on the source of the task-irrelevant object locations. The right precentral gyrus, extending to the right SMA, was generally activated by Simon conflicts, irrespective of the spatial reference frame involved, and showed no additive activity to Simon conflicts. In contrast, the right postcentral gyrus was specifically involved in Simon conflicts induced by task-irrelevant allocentric, rather than egocentric, representations. Furthermore, a right lateral frontoparietal network showed increased neural activity whenever the egocentric and allocentric target locations were incongruent, indicating its functional role as a mismatch detector that monitors the discrepancy concerning allocentric and egocentric object locations.

The human brain codes objects that are present in a visual scene simultaneously and in different spatial coordinate systems. For example, the spatial location of an object can be represented in an egocentric frame, i.e., its position is encoded with reference to the body effectors of the observer. Alternatively, an object's location can be represented in an allocentric frame, i.e., relative to an object or a background, independent of its egocentric position relative to the observer, such as the right or left side of an object in the outside world. These different types of representations constitute the basis for specific subsequent cognitive processes. In particular, egocentric representations are proposed to underlie goal-directed actions ${ }^{1-4}$, while allocentric representations are proposed to support conscious perception of objects or spatial memory functions $s^{1,2,5,6}$.

Accumulating previous evidence indicated that an object's location is automatically coded and cannot be ignored irrespective of whether or not it is irrelevant to a current behavioral task ${ }^{7}$. For example, in the classical Simon effect, subjects are requested to perform a discrimination task about a non-spatial object feature, such as color, luminance, shape, or sound, of a target, and indicate the respective feature by a manual response. In particular, a single feature is consistently mapped to either a left or a right manual response ${ }^{8-10}$. Although the egocentric position of the object (left/right) is irrelevant to the non-spatial discrimination task, it influences task

${ }^{1}$ Center for Studies of Psychological Application and School of Psychology, South China Normal University, Guangzhou, 510631, China. ${ }^{2}$ Cognitive Neuroscience, Institute of Neuroscience and Medicine (INM-3), Research Center Jülich, 52425, Jülich, Germany. ${ }^{3}$ Department of Neurology, University Hospital Cologne, 50937, Cologne, Germany. ${ }^{4}$ Guangdong Key Laboratory of Mental Health and Cognitive Science, South China Normal University, Guangzhou, 510631, P.R. China. Correspondence and requests for materials should be addressed to Q.C. (email: qi.chen27@gmail.com) 
performance depending on whether or not the target position is spatially consistent with the side of the response. Responses are slower when the irrelevant egocentric position of the target is incompatible with the side of the pre-defined manual response and quicker when both are compatible. This Simon effect has been observed across a variety of experimental manipulations, including the type of stimulus (e.g., auditory and visual stimulus ${ }^{11}$ ), spatial arrangement (e.g., vertical and horizontal presentation ${ }^{12}$ ), and reaction mode (e.g., cross hand response ${ }^{13}$; vocal response $\left.{ }^{14}\right)$. Neural mechanisms underlying the egocentric Simon effect have been found in the frontal-striatal cortex, anterior cingulate cortex (ACC), the pre-supplementary and the supplementary motor areas (pre-SMA/ SMA), and superior as well as inferior parietal gyrus (IPG) ${ }^{15-20}$. Interestingly, growing evidence suggests that the Simon effect is not only based on egocentric but also on allocentric coordinates ${ }^{10,21}$. For instance, responses are slower when the allocentric location of the target - although completely task-irrelevant - and the side of response hand are incongruent compared to when both are congruent. Behaviorally, the allocentric Simon effect has been demonstrated to be independent of the egocentric Simon congruency ${ }^{10}$.

It remains to be investigated, whether the neural mechanisms underlying the allocentric Simon effect are different from those involved in generating its egocentric variant or whether both share common resources. In the current $\mathrm{fMRI}$ study, to answer this question, we orthogonally combined the Simon effects based on the egocentric and allocentric reference frames with a non-spatial luminance discrimination task (Fig. 1A). The allocentric position of the behavioral target could be either consistent or inconsistent with the side of the response hand, irrespective of the spatial congruency between the target's egocentric position and the side of response hand. Therefore, the current factorial design resulted in four experimental conditions: (1) an egocentric congruent and allocentric congruent condition (EgoC_AlloC); (2) an egocentric congruent and allocentric incongruent condition (EgoC_AlloIC); (3) an egocentric incongruent and allocentric congruent condition (EgoIC_AlloC); and (4) an egocentric incongruent and allocentric incongruent condition (EgoIC_AlloIC) (Fig. 1A). This factorial design allows determining the main effects of spatial congruency within each reference frame, and hence localizing the neural mechanisms of the Simon effect induced within different reference frames. Furthermore, the factorial design allows revealing the neural interactions between the two types of Simon effects.

Brain regions that play a role in resolving conflicts induced by the Simon task are expected to show increased activation related to incongruent stimulus-response mappings, independent of whether these were induced by allocentric or egocentric coding of object locations. Furthermore, we were interested in two possible patterns of interaction between the neural processes underlying the egocentric and allocentric Simon effect. First, if the two types of Simon effect occur based on discrete spatial coordinate systems specific to the allocentric and egocentric representations, the two concrete conflict signals originating from the two specific spatial coordinate systems should be additive (Fig. 1B, left) ${ }^{22-24}$. Accordingly, neural activity in the double-source incongruent (EgoIC_ AlloIC) condition should be additive, i.e., equal to the summed activity of the two single-source incongruent (EgoIC_AlloC and EgoC_AlloIC) conditions. Alternatively, the two types of Simon conflict could occur based on a common higher-level or abstract spatial coordinate system, in which the allocentric and egocentric positions might be coded in an integrated fashion within the same abstract coordinate system (Fig. 1B, right). Since both share an abstract coordinate map, the conflict signal in the double-source incongruent (EgoIC_AlloIC) condition would be generated based on the same abstract incongruent coordinate as that in the two single-source incongruent conditions. Therefore, incongruent neural activity should be comparable between the three incongruent conditions.

More critically, in the congruent condition (EgoC_AlloC) and the double-source incongruent condition (EgoIC_AlloIC), the allocentric and egocentric positions of the target were on the same side, i.e., matched (Fig. 1A), while in the two single-source incongruent conditions (i.e., EgoC_AlloIC and EgoIC_AlloC), the allocentric and egocentric positions of the target were on the opposite side, i.e., mismatched (Fig. 1A). We accordingly hypothesized that, in the present study, there should be specific brain mechanisms involved in monitoring the mismatch of spatial representations about different spatial reference frames, rather than resolving the Simon conflict per se. In particular, the mismatch monitor should be significantly activated by the two single-source incongruent conditions (EgoC_AlloIC and EgoIC_AlloC), in which the egocentric and allocentric locations mismatched, compared with the congruent (EgoC_AlloC) and the double-source incongruent (EgoIC_ AlloIC) condition, in which the two sources of spatial representations matched.

\section{Materials and Methods}

Participants. Forty right-handed healthy volunteers (22 females and 18 males, $18-25$ years old) with no history of neurological, psychiatric, or color vision impairments took part in the current fMRI study. They all had a normal or corrected-to-normal vision. The study was designed and conducted following the guidelines of the Helsinki Declaration. All participants had given informed consent for the study before the experiment and got paid for their participation after the experiment. The Ethics Committee of the School of Psychology, South China Normal University, had approved the experimental procedures.

Stimuli and experimental setup. The target stimulus was a fork ( $2.5^{\circ}$ of visual angle in width $)$, placed on the top of an orange plate ( $15^{\circ}$ of visual angle in diameter), with a gray background (Fig. $\left.1 \mathrm{~A}\right)$. The color of the fork was either light (RGB value: $64,64,64$ ) or dark (RGB value: 192, 192, 192) gray in each trial. As shown in Fig. 1A, the fork had 4 allocentric positions with reference to the median sagittal axis of the plate (i.e., $-3.6^{\circ},-2^{\circ}, 2^{\circ}$, and $3.6^{\circ}$ ) and 4 egocentric positions with reference to the median sagittal axis of the participants' body (i.e., $-2.67^{\circ}$, $-1.7^{\circ}, 1.7^{\circ}$, and $2.67^{\circ}$ ) (Fig. $1 \mathrm{~A}$ ). The egocentric locations of the fork were orthogonally varied to the allocentric locations. The visual angles of the egocentric and allocentric positions of the target were chosen using a psychophysical test before the formal experiment, to balance the difficulty between the two judgment tasks. 
(A)

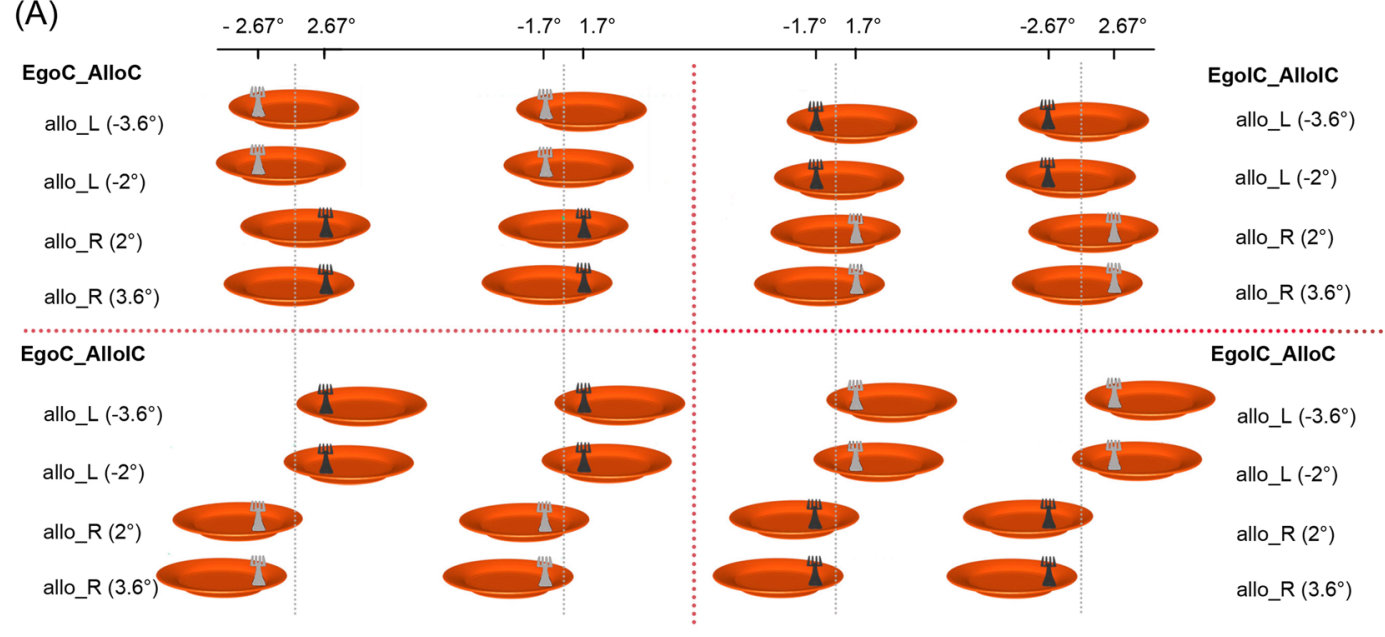

(B)

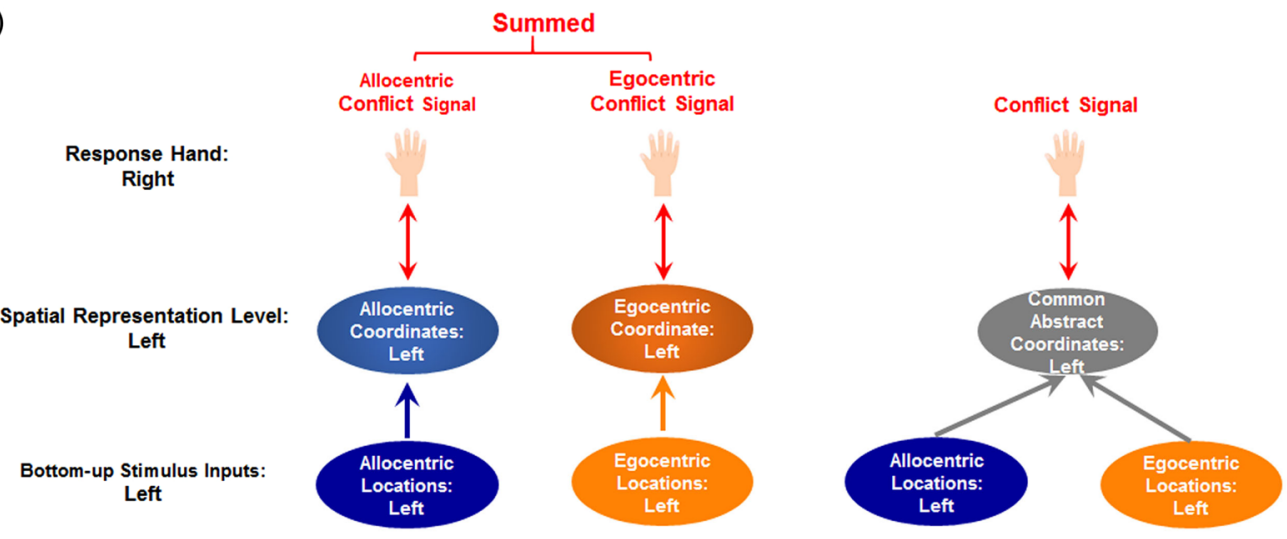

(C)

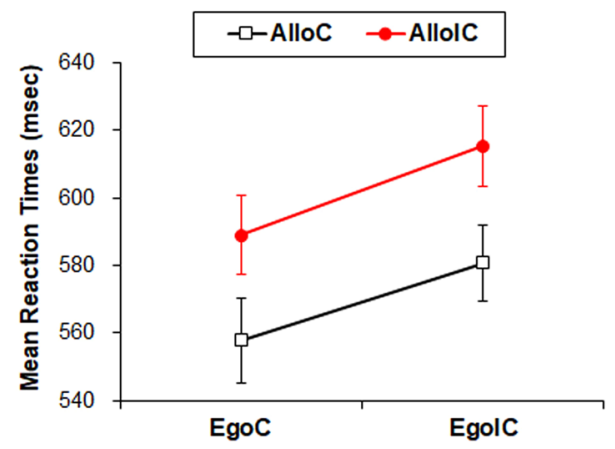

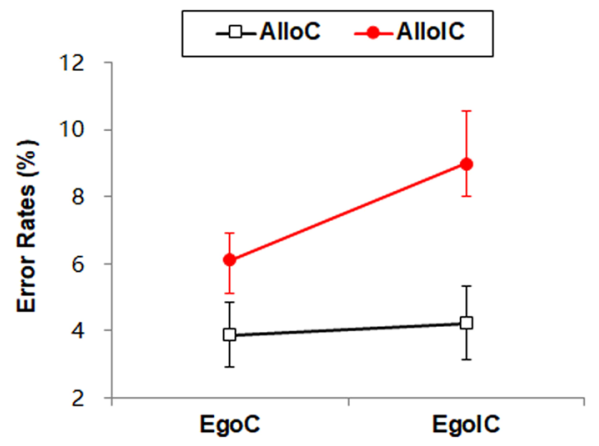

Figure 1. (A) Experimental stimuli. The target stimulus is the fork, with two levels of luminance. The gray dotted line represents the invisible egocentric mid-sagittal plane. The four egocentric locations $\left(-2.67^{\circ},-1.7^{\circ}\right.$, $\left.1.7^{\circ}, 2.67^{\circ}\right)$ of the fork are marked on the top of the figure, and the four allocentric locations $\left(-3.6^{\circ},-2^{\circ}, 2^{\circ}\right.$, $3.6^{\circ}$ ) of the fork (with reference to the mid-sagittal plane of the plate) are marked on the left and right side of the figure. For the examples of the four experimental conditions defined here, participants were asked to respond to the light grey via their left hand, and to the dark grey via their right hand. The bottom-up stimuli are fully counter-balanced for the three critical contrasts in the present experiment, i.e., the main effect of egocentric congruency "EgoC (AlloC + AlloIC) vs. EgoIC (AlloC + AlloIC)", the main effect of allocentric congruency "AlloC (EgoC + EgoIC) vs. AlloIC (EgoC + EgoIC)", and the interaction contrast "EgoC (AlloC > AlloIC) vs. EgoIC (AlloC > AlloIC)". (B) Two hypothetical models of the neural interaction between the egocentric and allocentric Simon effect. (C) Behavioral results. Mean RTs (ms) and mean error rates (\%) with standard errors in the four experimental conditions.

Experimental design and tasks. It has been suggested that participants tended to use the egocentric reference frame even when they were not explicitly instructed which specific frame to adopt ${ }^{25}$. Previous evidence from our lab further suggested that the allocentric Simon effect occurred only when the current task demands explicitly induced allocentric representations while the egocentric Simon effect existed irrespective of whether 
the egocentric representations were explicitly coded or not ${ }^{10}$. Therefore, to make sure that both the egocentric and allocentric Simon effects occur in the present experiment, we adopted two additional spatial judgment tasks (i.e., egocentric and allocentric judgment tasks), and alternated them with the main luminance discrimination task, to explicitly elicit the coding of the egocentric and allocentric representations. Specifically speaking, subjects were asked to perform three tasks on the same stimuli. First, participants were required to judge whether the position of the target was left or right respect to the median sagittal axis of their own body (egocentric judgment). Second, subjects were asked to judge whether the position of the target was left or right respect to the median sagittal axis of the plate (allocentric judgment task). Third, participants needed to discriminate whether the luminance type of the target was light or dark (luminance discrimination task). Currently, we focused our analysis on the non-spatial luminance discrimination tasks. Two response pads on each side of the participants' body were used. Participants were required to press a button on the left pad using their left thumb corresponding to the left side judgment or the dark gray judgment, and vice versa. The mapping between the luminance type and the response side was counterbalanced across all participants. The spatial judgment tasks and the non-spatial judgment tasks were alternated on a block-by-block basis. A luminance discrimination block followed every spatial judgment block. An instruction was displayed before each block for 3 seconds, informing the participants of the task in the upcoming block.

Moreover, in the luminance task, we manipulated the congruency between the task-irrelevant egocentric and allocentric positions of the target and the side of the response. The two types of the task-irrelevant spatial position of the fork could be either congruent or incongruent with the response side. Therefore, the experimental design about the luminance discrimination task was a 2 (egocentric congruency: incongruent vs. congruent) $\times 2$ (allocentric congruency: incongruent vs. congruent) within-subject design. The luminance discrimination task had 12 blocks. Each block contained 16 experimental trials and 5 to 6 null trials (only a blank screen was displayed). Within each block, the order of trials was randomized for each participant to avoid potential expectations. The target was presented for $250 \mathrm{~ms}$ in each trial. We chose such a short duration for minimizing unnecessary eye movements ${ }^{26}$. During the whole experiment, participants were asked to keep their eyes looking straightforward without moving them. The duration of each trial was jittered from $2000 \mathrm{~ms}$ to $3000 \mathrm{~ms}$ with a step of $250 \mathrm{~ms}$.

Data acquisition. All MR images were acquired by a $3 \mathrm{~T}$ Siemens Trio system with a standard 32-channel head coil. The following scanning parameters were used for T2*-weighted echo-planar images (EPI): $\mathrm{TR}=2.2 \mathrm{~s}$, $\mathrm{TE}=30 \mathrm{~ms}, \mathrm{FA}=90^{\circ}, \mathrm{FOV}=200 \mathrm{~mm}$, matrix $=64 \times 64$, voxel size $=3.4 \times 3.4 \times 3 \mathrm{~mm}^{3}$. We acquired 36 transversal slices covering the whole brain with a $0.75 \mathrm{~mm}$ gap. The functional scanning session resulted in $636 \mathrm{EPI}$ volumes, which lasted for 23.32 minutes. After the functional scanning, high-resolution images (voxel size $=1 \times 1 \times 1 \mathrm{~mm}^{3}$ ) of the whole brain were acquired for anatomic co-registration, using a standard T1-weighted 3D sequence (MPRAGE).

Statistical analysis of behavioral data. For each experimental condition, trials with incorrect responses or with reaction times (RTs) outside three times standard deviation (SD) were excluded from further analysis. Error rates were the percentage of excluded trials under each experimental condition. Mean RTs and error rates were then entered into a 2 (egocentric congruency: incongruent vs. congruent) $\times 2$ (allocentric congruency: incongruent vs. congruent) repeated-measures analyses of variance (ANOVA), respectively.

Statistical analyses of imaging data. All image data were processed and analyzed using the general linear model (GLM) in SPM12 (Wellcome Department of Imaging Neuroscience, London, http://www.fil.ion.ucl. ac.uk). First, the first 5 volumes were deleted, and the remaining images were realigned to the new first volume to correct for inter-scan head movement. Then, images were normalized to the standard MNI space and resampled to $2 \times 2 \times 2 \mathrm{~mm}^{3}$ voxel size. Finally, the normalized images were smoothed $(8 \mathrm{~mm}$ FWHM) to increase the signal/ noise ratio in the images. Data were high-pass-filtered at $1 / 200 \mathrm{~Hz}$.

At the first level, the GLM was used to construct a multiple regression design matrix. The four critical experimental conditions in the luminance discrimination tasks were modeled: "EgoC_AlloC", "EgoC_AlloIC", "EgoIC_ AlloC", and "EgoIC_AlloIC". Another two regressors were additionally modeled in the GLM, accounting for variances induced by all the trials in the egocentric and allocentric spatial judgment tasks, respectively, which were independent of the four critical regressors in the non-spatial luminance discrimination tasks. All the event types were time-locked to the onset of the target of each trial by a standard HRF and its first-order time derivative (TD) with an event duration of $0 \mathrm{~s}$. All the instructions, the error trials, and the six head movement parameters derived from the realignment procedure were included as covariates of no interest. Temporal autocorrelation was modeled using an AR (1) process. Parameter estimates were calculated for each voxel using weighted least-squares to provide maximum likelihood estimators based on the temporal autocorrelation of the data. For each participant, simple main effects for each of the 4 experimental conditions were computed by applying appropriate ' 10 ' baseline contrasts (experimental conditions vs. null trials). At the second group level, the 4 first-level individual contrast images were entered into a $2 \times 2$ within-subject ANOVA by employing a random-effects model. In the modeling of variance components, we allowed for violations of sphericity by modeling non-independence across parameter estimates from the same participant and allowed for unequal variances both between conditions and between participants using the standard implementation in SPM12.

First, we performed the conventional ANOVA analysis on the present factorial design, i.e., the main effect of allocentric congruency ["AlloIC (EgoC + AlloIC) > AlloC (EgoC + AlloIC)", and vice versa], the main effect of egocentric congruency ["EgoIC (AlloC + AlloIC) $>$ EgoC (AlloC + AlloIC)", and vice versa], and the interaction between allocentric and egocentric congruency ["EgoC (AlloIC > AlloC) > EgoIC (AlloIC > AlloC)", and vice versa]. The main effect contrasts are supposed to localize the specific neural activations involved in the congruency effect concerning one spatial reference frame, irrespective of the spatial congruency in the other 
reference frame. However, the present results showed that the allocentric and the egocentric Simon effect in the frontoparietal network could not be cleanly teased apart by the conventional analysis of the neural main effects (Fig. 2A). Rather, the significant main effect of the allocentric congruency seemed to be driven by an interaction effect (Fig. 2A): neural activity significantly increased in the ALLO_IC, compared to the ALLO_C condition, only in the EGO_C condition, but not in the EGO_IC condition. Therefore, the conventional analysis of main effects would not allow us to precisely localize the specific neural mechanisms underlying the allocentric vs. egocentric congruency effect. To further isolate the specific neural correlates underlying the different types of Simon conflicts, we directly compared the three incongruent conditions and localized the cortical regions that showed significantly higher neural activity in one incongruent condition, compared to the other two incongruent conditions. Specifically, "(EgoC_AlloIC $) * 2>$ [(EgoIC_AlloC $)+($ EgoIC_AlloIC $)]$ ” was calculated to localize the single-source allocentric Simon conflict, "(EgoIC_AlloC) $* 2>[($ EgoC_AlloIC $)+($ EgoIC_AlloIC) $]$ " was calculated to localize the single-source egocentric Simon conflict, and "(EgoIC_AlloIC) * $2>$ [(EgoC_ AlloIC) + (EgoIC_AlloC)]" was calculated to localize the double-source Simon conflict induced by both frames.

Moreover, to localize the common neural correlates between the three types of Simon conflicts, we first calculated the specific pattern of neural activity elicited by the three incongruent conditions, as compared to the congruent condition, i.e., "EgoC_AlloIC > EgoC_AlloC", "EgoIC_AlloC > EgoC_AlloC", and "EgoIC_ AlloIC > EgoC_AlloC". Subsequently, a statistical conjunction was performed between the above three contrasts, i.e., "(EgoC_AlloIC $>$ EgoC_AlloC $) \cap($ EgoIC_AlloC $>$ EgoC_AlloC $) \cap($ EgoIC_AlloIC $>$ EgoC_AlloC)". The conjunction null hypothesis, instead of the global null hypothesis, was tested for the conjunction analyses ${ }^{18,27}$. For all statistical analyses, areas of activation were identified as significant only if they passed a threshold of $\mathrm{p}<0.001$, family-wise error (FWE) correction for multiple comparisons at the cluster level, with an underlying voxel level of $\mathrm{p}<0.001$ uncorrected $^{28}$.

Three regions of interest (ROI) were defined based on the results of group analysis: (1) the right precentral gyrus (PreCG; MNI: 42, -10, 56; BA6), which was generally activated by the Simon conflicts "(EgoC_ AlloIC $>$ EgoC_AlloC $) \cap($ EgoIC_AlloC $>$ EgoC_AlloC $) \cap$ (EgoIC_AlloIC $>$ EgoC_AlloC)”, no matter which spatial reference frame was involved (Fig. 3A and Table 2A); (2) the right postcentral gyrus (PosCG; MNI: 48, $-28,50$; BA2), which was specifically activated by the allocentric Simon conflict "(EgoC_AlloIC) *2> [(EgoIC_ AlloC) +EgoIC_AlloIC)]" (Fig. 3B and Table 2B); and (3) the posterior portion of the right superior parietal gyrus (posterior SPG; MNI: 24, -70, 48; BA7), which was involved in the neural interaction contrast "EgoC (AlloIC $>$ AlloC) $>$ EgoIC (AlloIC $>$ AlloC)" (Fig. 2B and Table 1B). In the three ROIs, mean parameter estimates of the critical condition were further extracted using MarsBar 0.44 (http://sourceforge.net/projects/marsbar). No further ANOVA was performed on the extracted parameter estimates to avoid the problem of double dipping ${ }^{29,30}$. The figures of the extracted parameter estimates in Figs 2 and 3 were drawn only for demonstration purposes.

Psychophysiological interaction (PPI) analysis. According to the results of neural interaction contrast "EgoC (AlloIC > AlloC) > EgoIC (AlloIC > AlloC)", the activation of the right SPG significantly increased when the egocentric and allocentric position mismatched (i.e. one left and one right) in the EgoC_AlloIC and EgoIC_ AlloC conditions than when matched (i.e. both left or both right) in the EgoC_AlloC and EgoIC_AlloIC conditions (Fig. 2B and Table 1B). To further investigate how the right SPG was involved in monitoring the "match vs. mismatch" in spatial locations relative to the two spatial reference frames, the right posterior SPG was used as a source region (MNI: 24, $-70,48$; Fig. 2B and Table 1B) to estimate the context-specific functional modulation of neural activity across the brain, using PPI analysis. The neural activity in the right SPG was used as the physiological factor, and the contrast "Mismatch (EgoC_AlloIC + EgoIC_AlloC) vs. Match (EgoC_AlloC+EgoIC_AlloIC)", which is identical to the neural interaction contrast, was used as the psychological factor.

First, the neural contrast "Mismatch $>$ Match" was calculated at the individual level. Then, individual peak voxel was determined as the maximally activated voxel within a sphere of $16 \mathrm{~mm}$ radius (i.e., twice the smoothing kernel) around the peak voxel within the right posterior SPG (MNI: 24, -70, 48) for each participant. All individual peak voxels were located in the right SPG $(x=24 \pm 8, y=-70 \pm 6, z=48 \pm 6)$. Next, time series were extracted from a sphere of $4 \mathrm{~mm}$ radius (twice the voxel size) around the individual peak voxels within the right SPG. PPI analysis at the individual level employed three regressors: (1) The physiological variable of interest (i.e. the time series extracted from the right SPG); (2) The psychological variable of interest (i.e. "Mismatch vs. Match"), (3) The cross product of the previous two (i.e. the PPI term). An SPM was calculated to reveal brain areas in which the neural activation was predicted by the PPI term, with the physiological and the psychological regressors being treated as confound variables, i.e., by putting 1 on the PPI regressor and 0 on the physiological and the psychological regressors, respectively. At the group level, the random-effects analysis was adopted: the individual SPMs corresponding to the PPI term of each participant were subsequently fed into a one-sample $t$-test $(p<0.001$, FWE correction at the cluster level, with an underlying voxel level of $p<0.001$, uncorrected).

\section{Results}

Behavioral results. For RTs, the main effect of egocentric congruency was significant, $F_{(1,39)}=32.95$, $p<0.001$, indicating that participants responded significantly slower in the EgoIC condition $(598 \pm 11 \mathrm{~ms})$ than in the EgoC $(573 \pm 12 \mathrm{~ms})$ condition, i.e., a significant Simon effect based on egocentric locations. The main effect of allocentric congruency was significant as well, $F_{(1,39)}=62.72, \mathrm{p}<0.001$, indicating that participants responded significantly slower in the AlloIC condition $(602 \pm 11 \mathrm{~ms})$ than in the AlloC condition $(569 \pm 12 \mathrm{~ms})$, i.e., a significant Simon effect based on allocentric locations. The two-way interaction was not significant, $F_{(1,39)}<1$ (Fig. 1C, left).

For error rates, the pattern of results was similar to that of RTs. The main effect of egocentric congruency was significant, $F_{(1,39)}=4.13, p<0.05$, with participants making more errors in the EgoIC condition $(6.6 \% \pm 1 \%)$ than in the EgoC condition $(5.0 \% \pm 0.9 \%)$. The main effect of allocentric congruency was also significant, 
(A) Main Effect of the Allocentric Congruency
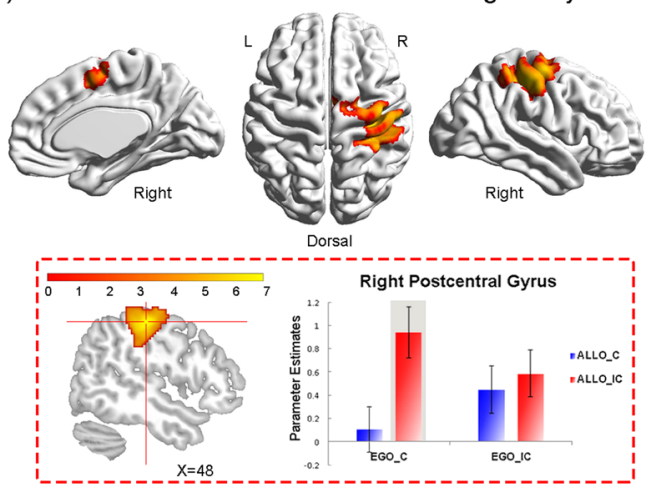

(B)

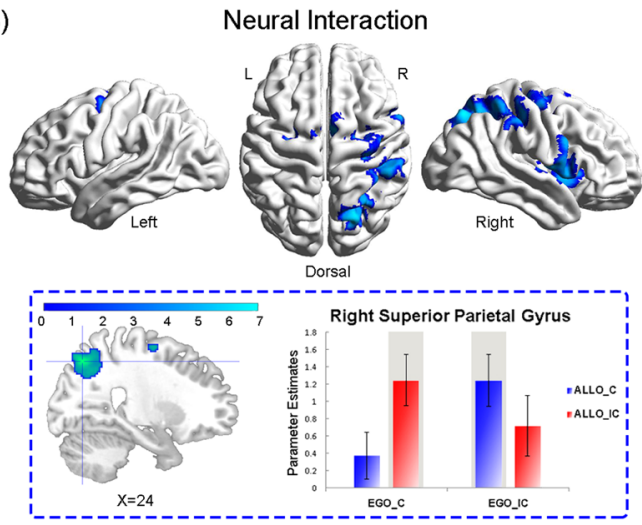

(C)

Psychophysiological Interaction

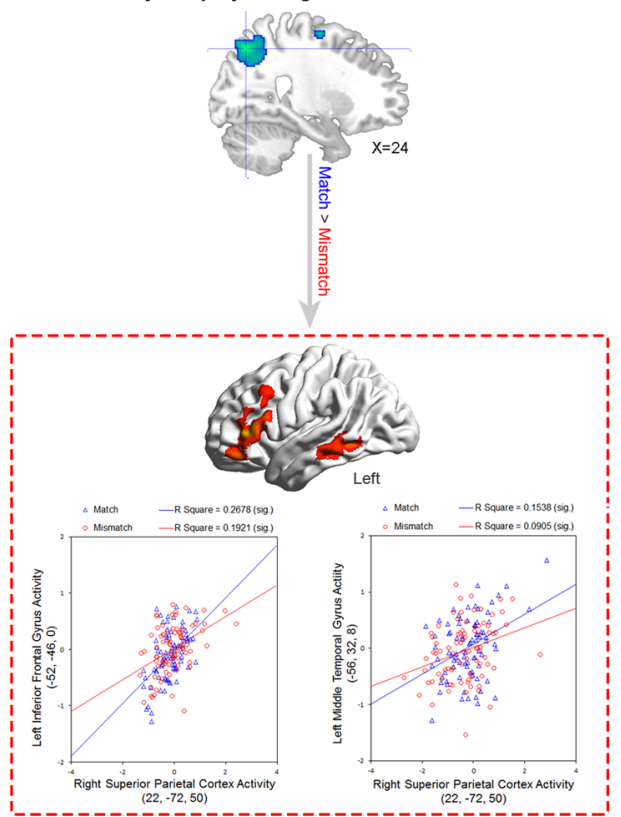

Figure 2. (A) Main effect of the allocentric congruency. Brain areas activated by allocentric incongruent conditions relative to the allocentric congruent conditions irrespective of the egocentric congruency, i.e., AlloIC $($ EgoC + EgoIC $)>$ AlloC $($ EgoC + EgoIC $)$. Mean parameter estimates extracted from the activated cluster are shown as a function of the four experimental conditions. (B) Neural interactions between the egocentric and the allocentric congruency, i.e. the neural interaction contrast "EgoC (AlloIC > AlloC) > EgoIC (AlloIC > AlloC)". Mean parameter estimates extracted from the activated cluster are shown as a function of the four experimental conditions. Error bars indicate SEs. (C) PPI analysis based on neural activity in the right SPG (MNI: 24, -70 , 48 ) with the contrast "Match vs. Mismatch" as the psychological factor. The right SPG (upper panel) showed enhanced functional connectivity with the left IFC and the left MT (lower panel) in the "Match" conditions than in the "Mismatch" conditions. For a representative participant, mean corrected neural activity in the left IFC (lower left panel) and the left MT (lower right panel) was plotted as a function of the mean corrected neural activity in the right SPG in the "Match" and "Mismatch" conditions, respectively. 


\section{(A) Common Neural Activity of Simon Conflicts}
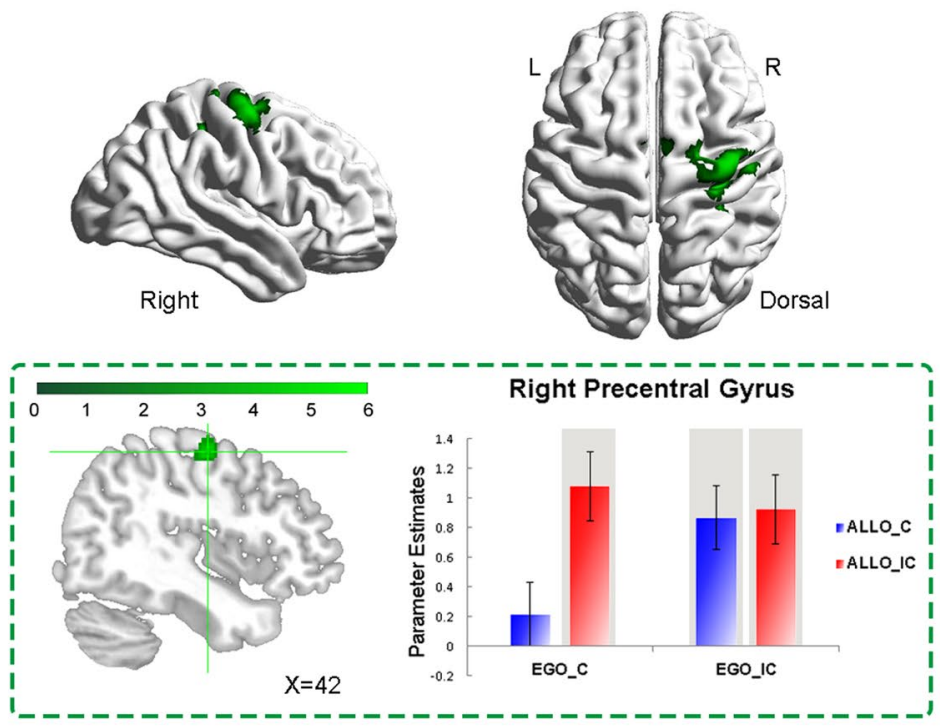

\section{(B) Specific Neural Activity of Allocentric Simon Effect}
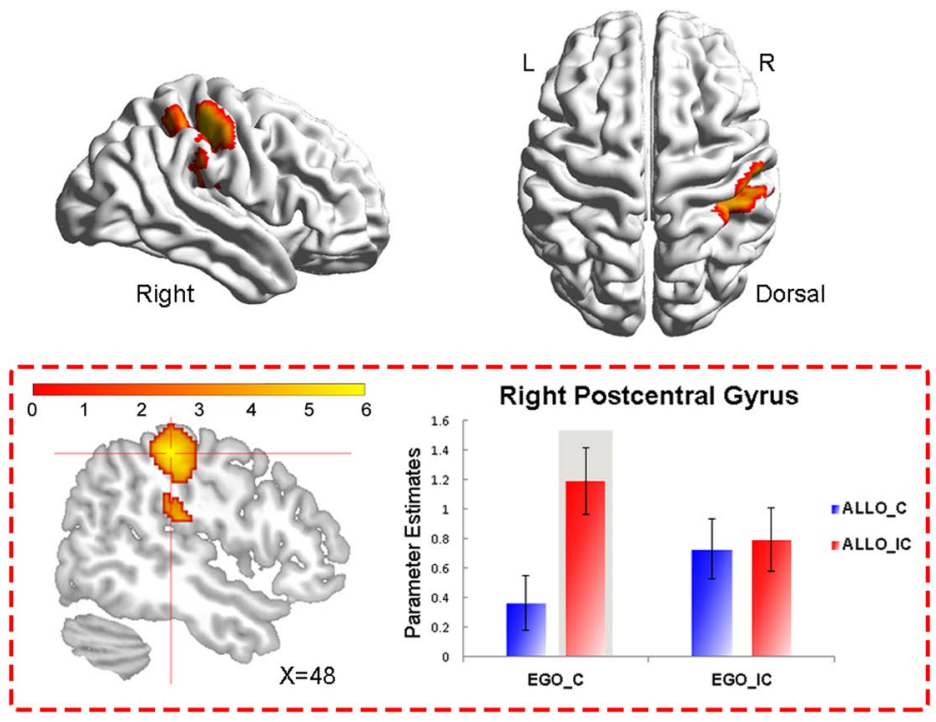

(C)

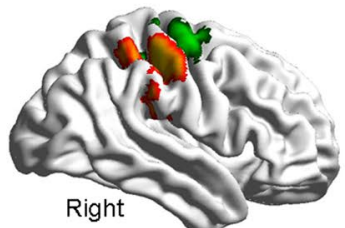

- Common Neural Activity of Simon Conflicts

- Specific Neural Activity of Allocentric Simon Effect

Figure 3. (A) Common neural correlates underlying the single-source and the double-source incongruent conditions. The right precentral gyrus was activated by the conjunction analysis between the singlesource allocentric conflict, i.e., EgoC (AlloIC > AlloC), the single-source egocentric conflict, i.e., AlloC (EgoIC $>$ EgoC), and the double-source conflict, i.e., EgoIC_AlloIC $>$ EgoC_AlloC. Mean parameter estimates extracted from the activated cluster are shown as a function of the four experimental conditions. (B) Specific neural correlates underlying the allocentric Simon conflict. Parameter estimates were extracted from the activated clusters in the right PosCG, and are shown as a function of the four experimental conditions. Error bars indicate SEs. (C) Overlay of the activated brain regions in (A) (green) and (B) (orange).

$F_{(1,39)}=12.51, p<0.01$, with participants making more errors in the AlloIC $(7.6 \% \pm 1.2 \%)$ condition than in the AlloC $(4.1 \% \pm 0.7 \%)$ condition. The interaction between allocentric and egocentric congruency was not significant, $F_{(1,39)}=2.65, p=0.11$ (Fig. $1 \mathrm{C}$, right). 


\begin{tabular}{|c|c|c|c|c|}
\hline Anatomical region & Side & $\begin{array}{l}\text { Cluster peak } \\
(\mathbf{m m})\end{array}$ & $t$-Score & $k E$ (voxels) \\
\hline \multicolumn{5}{|c|}{ (A) AlloIC $($ EgoC + EgoIC $)>$ AlloC $($ EgoC + EgoIC $)$} \\
\hline Postcentral gyrus & $\mathrm{R}$ & $48,-22,52$ & 6.80 & 2776 \\
\hline Precentral gyrus & $R$ & $42,-10,58$ & 6.42 & \\
\hline \multicolumn{5}{|c|}{ (B) EgoC $($ AlloIC $>$ AlloC $)>$ EgoIC $($ AlloIC $>$ AlloC $)$} \\
\hline Superior parietal gyrus & $\mathrm{R}$ & $24,-70,48$ & 5.46 & 1911 \\
\hline Inferior parietal gyrus & $R$ & $48,-32,50$ & 4.93 & \\
\hline Postcentral gyrus & $R$ & $44,-28,42$ & 4.25 & \\
\hline Supplementary motor area & $\mathrm{R}$ & $10,2,60$ & 5.32 & 566 \\
\hline Superior frontal gyrus & $L$ & $-16,6,52$ & 4.51 & \\
\hline Supplementary motor area & $L$ & $-10,4,52$ & 4.30 & \\
\hline Cerebellum & $\mathrm{L}$ & $-6,-66,-20$ & 5.20 & 383 \\
\hline Rolandic operculum & $\mathrm{R}$ & $52,-6,8$ & 4.74 & 875 \\
\hline Precentral gyrus & $R$ & $58,8,26$ & 4.63 & \\
\hline Superior frontal gyrus & $\mathrm{R}$ & $26,-6,62$ & 4.37 & 337 \\
\hline Precentral gyrus & $R$ & $34,-16,60$ & 4.33 & \\
\hline \multicolumn{5}{|l|}{ (C) PPI results } \\
\hline Inferior frontal gyrus & $\mathrm{L}$ & $-52,36,8$ & 6.64 & 612 \\
\hline Middle temporal gyrus & $\mathrm{L}$ & $-52,-46,0$ & 5.10 & 332 \\
\hline
\end{tabular}

Table 1. (A) Main effects of allocentric congruency. (B) The neural interaction between egocentric and allocentric congruency. (C) Brain regions that showed higher functional connectivity with the right superior parietal cortex in the "Match" than "Mismatch" condition. The coordinates (x, y, z) correspond to MNI coordinates. Displayed are the coordinates of the maximally activated voxel within a significant cluster as well as the coordinates of relevant local maxima within the cluster (in Italics).

\begin{tabular}{|c|c|c|c|c|}
\hline Anatomical region & Side & Cluster peak (mm) & $t$-Score & $k_{\mathrm{E}}$ (voxels) \\
\hline \multicolumn{5}{|c|}{$\begin{array}{l}\text { (A) Conjunction: }(\text { EgoC_AlloIC }>\text { EgoC_AlloC }) \cap(\text { EgoIC_AlloC }>\text { EgoC_ } \\
\text { AlloC }) \cap(\text { EgoIC_AlloIC }>\text { EgoC_AlloC })\end{array}$} \\
\hline Precentral gyrus & $\mathrm{R}$ & $42,-10,56$ & 5.19 & 1010 \\
\hline Precentral gyrus & $R$ & $30,-24,52$ & 4.54 & \\
\hline Postcentral gyrus & $R$ & $30,-36,46$ & 4.17 & \\
\hline Supplementary motor area & $R$ & $8,0,54$ & 4.12 & \\
\hline \multicolumn{5}{|c|}{ (B) $($ EgoC_AlloIC $) * 2>($ EgoIC_AlloC + EgoIC_AlloIC $)$} \\
\hline Postcentral gyrus & $\mathrm{R}$ & $48,-28,50$ & 5.97 & 937 \\
\hline Postcentral gyrus & $R$ & $42,-30,44$ & 5.21 & \\
\hline Inferior Parietal gyrus & $R$ & $36,-40,50$ & 4.27 & \\
\hline Supramarginal gyrus & $R$ & $36,-36,44$ & 4.14 & \\
\hline
\end{tabular}

Table 2. (A) Common neural activity underlying the Simon conflicts, irrespective of the spatial reference frame involved. (B) Specific conflict-related activity of the allocentric Simon effect. The coordinates (x, y, z) correspond to MNI coordinates. Displayed are the coordinates of the maximally activated voxel within a significant cluster as well as the coordinates of relevant local maxima within the cluster (in Italics).

Imaging results. Main effect of allocentric congruency. We first identified the brain regions activated by the main effect of allocentric congruency, i.e. "AlloIC (EgoC + EgoIC) > AlloC (EgoC + EgoIC)". The right PosCG, extending anteriorly to the right PreCG, was significantly activated by the main effect contrast "AlloIC $>$ AlloC" (collapsed over the factor of egocentric congruency; Fig. 2A and Table 1A). Based on the observed pattern of neural activity in the right PosCG (MNI: 48, -28, 50; Fig. 2A), the main effect of allocentric congruency in the right PosCG seems to be driven by the allocentric congruency effect only in the EgoC condition, but not in the EgoIC condition, i.e. an interaction rather than main effect. A conjunction analysis between the main effect of the allocentric congruency "AlloIC (EgoC + EgoIC) $>$ AlloC (EgoC + EgoIC)" and the interaction effect "EgoC $($ AlloIC $>$ AlloC) $>$ EgoIC (AlloIC $>$ AlloC)" further confirmed overlapping activations in the right PosCG (MNI: $48,-32,50 ; Z=4.69,532$ voxels), indicating that the significant main effect of allocentric congruency in the right PosCG is indeed driven by the interaction effect.

The reverse contrast, i.e., AlloC > AlloIC (collapsed over the factor of egocentric congruency), revealed no significant activation.

Main effect of egocentric congruency. No significant activation was revealed by the main effects of the egocentric congruency EgoIC vs. EgoC (collapsed over the factor of allocentric congruency). 
Neural interaction between the egocentric and the allocentric congruency. The neural interaction contrast "EgoC $($ AlloIC $>$ AlloC) $>$ EgoIC (AlloIC $>$ AlloC)" revealed significant activations in the right frontoparietal network, with maximum activation in the posterior portion of the right SPG (MNI: 24, -70, 48, BA7), extending dorsally to the right SMA and right superior frontal gyrus (SFG), and ventrally to the rolandic operculum (ROL) (Fig. 2B and Table 1B). No significant activations were observed in the reverse interaction contrast. Neural activity in the two single-source incongruent conditions (i.e., EgoC_AlloIC and EgoIC_AlloC) was both higher than in the congruent condition (i.e., EgoC_AlloC) and the double-source incongruent condition (i.e., EgoIC_AlloIC).

Since the allocentric and egocentric positions mismatched (i.e., one left and one right) in the EgoC_AlloIC and EgoIC_AlloC conditions, while matching (i.e., both left or both right) in the EgoC_AlloC and EgoIC_AlloIC conditions, the right SPG was involved in monitoring the mismatch in spatial locations relative to the egocentric and allocentric reference frame. To further investigate how the SPG communicates with the other brain regions as a "match vs. mismatch" detector, we further performed a PPI analysis with the interaction contrast "Mismatch (EgoC_AlloIC + EgoIC_AlloC) vs. Match (EgoC_AlloC + EgoIC_AlloIC)" as the psychological factor and with neural activity in the right SPG as the physiological factor. The right SPG showed significantly higher functional connectivity with the left middle temporal gyrus (MT) and the left ventral lateral inferior frontal gyrus (IFG) in the "Match" conditions (i.e., EgoC_AlloC and EgoIC_AlloIC) than in the "Mismatch" conditions (i.e., EgoC_ AlloIC and EgoIC_AlloC) (Fig. 2C; Table 1C).

No significant modulation of neural coupling was found in the reverse direction (i.e., "Mismatch $>$ Match"). The PPI analysis calculated functional connections between different brain areas, regardless of the height of neural activity. Therefore, even though the right SPG showed higher neural activity in the "Mismatch" than "Match" condition, the neural coupling between the right SPG and the ventral visual stream and the ventral lateral IFG was higher in the "Match" than "Mismatch" conditions.

Common neural correlates underlying the egocentric and allocentric Simon effects. Compared to the congruent condition (EgoC_AlloC), the single-source allocentric Simon conflict condition (EgoC_AlloIC) activated a dorsolateral frontoparietal network, including the bilateral posterior SPG, the bilateral IPG, the right PreCG, and the right PosCG (Fig. 4A and Table 3A). The single-source egocentric Simon conflict condition (EgoIC_AlloC) activated a similar frontoparietal network including the right SPG, the right angular gyrus (ANG), the right PreCG, and the right SMA (Fig. 4B and Table 3B). The double-source incongruent condition (EgoIC_AlloIC) activated a relatively smaller brain region, including the right PreCG and the right PosCG (Fig. 4C and Table 3C).

To isolate the cortical regions that generally respond to the allocentric and egocentric Simon conflict whenever there is a conflict component irrespective of the spatial reference frame involved, a conjunction analysis was performed between the three incongruent conditions, i.e., the allocentric Simon conflict "EgoC (AlloIC $>$ AlloC)" (Fig. 4A), the egocentric Simon conflict "AlloC (EgoIC > EgoC)" (Fig. 4B), and the Simon conflict based on both frames "EgoIC_AlloIC > EgoC_AlloC" (Fig. 4C). One cluster, extending from right PreCG to right SMA with maximum activation in the right PreCG, was significantly activated (Fig. 3A and Table 2A). Neural activity was significantly higher in all three incongruent conditions as compared to the congruent condition. Moreover, neural activity between the three incongruent conditions was comparable. The above results suggested that the right PreCG generally responded to the Simon effect irrespective of the spatial reference frame involved. Furthermore, since the height of neural activity in the double-source incongruent condition (i.e., EgoIC_AlloIC) was comparable to that in the two single-source incongruent conditions (i.e., EgoC_AlloIC and EgoIC_AlloC), the right PreCG did not respond to the egocentric and allocentric Simon conflict in an additive way.

Specific neural correlates underlying the allocentric Simon effect. To further isolate the specific neural correlates underlying the allocentric Simon conflict, we directly compared the three incongruent conditions, and localized the cortical regions that showed significantly higher neural activity in the EgoC_AlloIC condition compared to the other two incongruent conditions, by using the neural contrast "(EgoC_AlloIC) *2 $>$ [(EgoIC_AlloC) + (EgoIC AlloIC)]". One cluster, extending from the right PosCG to the right IPG, was significantly activated (Fig. 3B and Table 2B). Neural activity was higher in the allocentric Simon conflict condition (EgoC_AlloIC) than in the other two incongruent conditions (EgoIC_AlloC and EgoIC_AlloIC).

In addition, the two neural contrasts "(EgoIC_AlloC) $* 2>[($ EgoC_AlloIC $)+($ EgoIC_AlloIC $)]$ " and "(EgoIC_AlloIC $) * 2>$ [(EgoC_AlloIC $)+($ EgoIC_AlloC) $]$ " were used to localize the specific neural correlates underlying the single-source egocentric Simon conflict and the double-source conflict induced by both frames, respectively. However, no significant activation was found.

\section{Discussion}

By orthogonally manipulating the Simon effect based on allocentric and egocentric spatial reference frames, in this fMRI study, we revealed novel neural correlates underlying the allocentric Simon effect and its neural interactions with the egocentric Simon effect. Behaviorally, both the classic egocentric Simon effect and the allocentric Simon effect were observed (Fig. 1C). Neurally, we found three different patterns of neural interactions between the allocentric and egocentric Simon effect (Figs 2 and 3). In the following paragraphs, we are going to focus our discussion on the three different patterns of neural interaction and the underlying theoretical implications.

General neural mechanisms underlying the Simon effect. The right PreCG, extending to the right SMA, showed significantly increased neural responses in all the three incongruent conditions, compared to the congruent condition (Fig. 3A), suggesting that these areas were generally activated by Simon conflicts, irrespective of the type of spatial reference frame involved. 

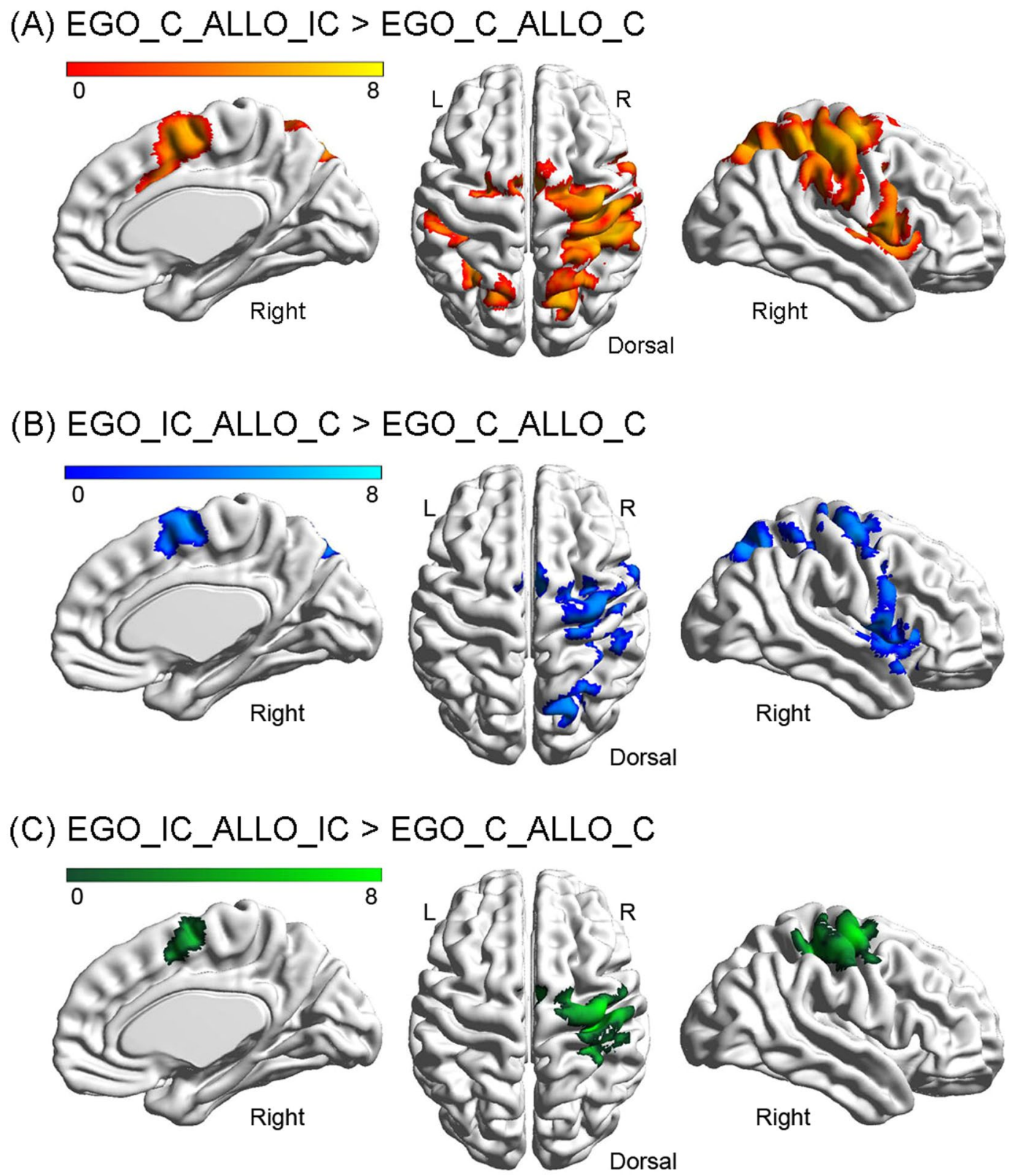

Figure 4. Neural activations in the three incongruent conditions as compared with the congruent condition. (A) Single-source allocentric incongruent condition, i.e., EgoC_AlloIC > EgoC_AlloC. (B) Single-source egocentric incongruent condition, i.e., EgoIC_AlloC $>$ EgoC_AlloC. (C) Double-source incongruent condition, i.e., EgoIC_AlloIC > EgoC_AlloC.

It has been reported that the PreCG was generally associated with various types of spatial compatibility tasks ${ }^{31-36}$. In particular, when participants were asked to respond to stimuli presented in either the right or left visual field with either the ipsilateral (compatible conditions) or the contralateral hand (incompatible conditions), the activation of PreCG significantly increased in the incompatible conditions as compared to the compatible conditions ${ }^{32,34}$. Furthermore, previous evidence has shown that single-pulse Transcranial Magnetic Stimulation (TMS) over the PreCG induced facilitatory responses in the incongruent condition, suggesting a causal-functional role of the PreCG in conflict resolution ${ }^{34}$. Similar to the PreCG, previous evidence from a large body of neuroimaging research on a variety of different conflict tasks, such as the Simon, Stroop, and Eriksen flanker tasks, points to a general role of the SMA in conflict resolution ${ }^{15,17,19,35-39}$. For example, it has been suggested that the SMA is commonly involved in both the Stroop and Simon conflicts ${ }^{15}$. Also, by asking subjects to identify shapes based on form-from-motion perception within a randomly moving dot field while ignoring the motion direction or stimulus location, Wittfoth (2006) found that the two variations of the Simon task shared neural activations in pre-SMA/SMA during conflict resolution ${ }^{19}$.

In the Simon-type tasks, the task-irrelevant position automatically activates the ipsilateral response through a direct route, whereas the task-relevant stimulus feature activates the correct response via a controlled route ${ }^{40}$. To successfully perform the current task, especially in the incongruent conditions, participants were required to override 


\begin{tabular}{|c|c|c|c|c|}
\hline Anatomical region & Side & Cluster peak $(\mathrm{mm})$ & $t$-Score & $k_{\mathrm{E}}$ (voxels) \\
\hline \multicolumn{5}{|c|}{ (A) EgoC_AlloIC $>$ EgoC_AlloC } \\
\hline Postcentral gyrus & $\mathrm{R}$ & $46,-32,52$ & 7.88 & 7530 \\
\hline Postcentral gyrus & $R$ & $48,-24,48$ & 7.72 & \\
\hline Precentral gyrus & $R$ & $42,-10,58$ & 7.50 & \\
\hline Rolandic operculum & $\mathrm{R}$ & $60,6,12$ & 6.08 & 1702 \\
\hline Insula lobe & $R$ & $40,0,12$ & 5.89 & \\
\hline Inferior parietal gyrus & $\mathrm{L}$ & $-30,-48,42$ & 5.67 & 1071 \\
\hline Superior parietal gyrus & $L$ & $-18,-62,56$ & 4.88 & \\
\hline Cerebellum & $\mathrm{L}$ & $-4,-68,-24$ & 6.21 & 725 \\
\hline \multicolumn{5}{|c|}{ (B) EgoIC_AlloC $>$ EgoC_AlloC } \\
\hline Supplementary motor area & $\mathrm{R}$ & $10,0,60$ & 5.66 & 1926 \\
\hline Precentral gyrus & $R$ & $42,-10,56$ & 5.19 & \\
\hline Insula lobe & $\mathrm{R}$ & $44,8,0$ & 4.54 & 964 \\
\hline Precentral gyrus & $R$ & $58,6,20$ & 4.37 & \\
\hline Superior parietal gyrus & $\mathrm{R}$ & $22,-68,50$ & 4.68 & 819 \\
\hline Angulargyrus & $R$ & $32,-58,52$ & 4.38 & \\
\hline Superior parietal gyrus & $R$ & $38,-48,58$ & 3.85 & \\
\hline Cerebellum & $\mathrm{L}$ & $-4,-66,-22$ & 6.6 & 774 \\
\hline \multicolumn{5}{|c|}{ (C) EgoIC_AlloIC $>$ EgoC_AlloC } \\
\hline Precentral gyrus & $\mathrm{R}$ & $34,-24,50$ & 6.71 & 2288 \\
\hline Postcentral gyrus & $R$ & $32,-34,48$ & 4.99 & \\
\hline
\end{tabular}

Table 3. Neural regions activated in the three incongruent conditions compared to the congruent condition. The coordinates $(\mathrm{x}, \mathrm{y}, \mathrm{z})$ correspond to MNI coordinates. Displayed are the coordinates of the maximally activated voxel within a significant cluster as well as the coordinates of relevant local maxima within the cluster (in Italics).

contextual influences on the motor system emerging from irrelevant target representations, within the egocentric or the allocentric reference frame or both, in order to maintain unbiased spatial representations of the response hand. The PreCG, comprised of premotor and primary motor area, is considered as a crucial neural area for planning, selecting, and executing responses ${ }^{41-45}$. Evidence from previous Simon type tasks suggests that the Simon conflict is associated with neural responses in the PreCG that are involved in inhibiting task-irrelevant representations ${ }^{46-52}$. On the other hand, the SMA is associated with executive control of motor outputs, in particular with selecting the appropriate response between different response alternatives ${ }^{53-55}$. In the current study, neural activations in the PreCG and SMA might be involved in voluntarily selecting the task-relevant correct responses while inhibiting the motor tendency induced by the task-irrelevant spatial positions. Taken together, the present results not only confirm but also extend previous evidence by showing that the right PreCG and the right SMA subserved the maintenance of unbiased response representations by resolving the conflicts from contextual information, irrespective of whether the contextual information is based on the allocentric or the egocentric reference frame.

Moreover, our results showed that the right PreCG and SMA do not respond to the Simon conflict in an additive way: the height of neural activity in the double-source incongruent (EgoIC_AlloIC) condition was comparable to that in the two single-source incongruent (EgoC_AlloIC and EgoIC_AlloC) conditions (Fig. 3A). Therefore, this pattern of results supports the model that the allocentric and egocentric Simon conflicts occur based on a common abstract spatial coordinate system in which the allocentric and egocentric positions are coded by the same abstract coordinate (Fig. 1B, right). It is widely accepted that spatial locations of stimuli are automatically encoded, even though they may be entirely irrelevant for an ongoing task ${ }^{56,57}$. In particular, the spatial location of an object could be automatically encoded upon its appearance for both the egocentric and the allocentric reference ${ }^{10,58-60}$. From an evolutionary point of view, an economical solution to simultaneously code the allocentric and egocentric locations of an object in the human brain is to use one common abstract coordinate, which allows different reference frames to converge onto a unified spatial coordinate system. Therefore, during sensorimotor transformation, spatial representations of discrete sensory stimuli are transformed into a common high-level abstract coordinate system ${ }^{5,61,62}$. In the double-source incongruent (EgoIC_AlloIC) condition of the present study, the egocentric and allocentric locations of the target share the same coordinate in the high-level abstract coordinate system (Fig. 1B right) even though that one is coded as left while the other is coded as right (Fig. 1A). The PreCG and SMA may be generally involved in inhibiting the incongruent spatial codes in the abstract coordinate system, rather than the discrete allocentric and egocentric representations per se. Since the single-source and the double-source incongruent conditions refer to the same abstract incongruent coordinates, it does not make any difference for the PreCG and SMA to inhibit the same abstract incongruent code between the two conditions.

Specific neural mechanisms underlying the allocentric Simon effect. The caudal part of the PosCG (BA2) was specifically involved in the allocentric Simon effect, by showing increased neural activity in the allocentric Simon conflict (EgoC_ AlloIC), compared to the egocentric Simon conflict (EgoIC_ AlloC), and the 
Simon conflict induced by both frames (EgoIC_ AlloIC) (Figs 2A and 3B). In the allocentric reference frame, object positions are primarily represented relative to the configurational properties of objects, such as the relationships among different components of one object or different objects in the environment. It has been demonstrated that allocentric spatial representations subserve the conscious perception/identification of objects and are represented mainly along the ventral visual stream ${ }^{3,63-68}$. In order to make goal-directed actions towards external objects, however, the discrete object representations in the ventral visual stream need to be transformed to the corresponding sensorimotor representations in the frontoparietal network via the dorsal visual stream ${ }^{69-71}$. Previous evidence from our lab showed that allocentric judgments on object locations activated both the ventral visual areas and the dorsal frontoparietal network ${ }^{72}$. Moreover, part of the right PosCG was involved in resolving spatial conflicts caused by task-irrelevant allocentric representations during egocentric judgment tasks ${ }^{60}$. In the current study, we further revealed that the right PosCG was specifically involved in the Simon type conflicts between the task-irrelevant allocentric representations and the response hand (Fig. 3B).

The PosCG (primary somatosensory cortex) is reported to be the confluence of several interconnected sensorimotor processes. These processes depend on visual, tactile, and motorial information to generate and execute goal-directed actions, such as visually guided reaching and grasping movements ${ }^{64,73-76}$. Moreover, previous neuroimaging studies on various types of the Simon effect suggest that the right postcentral area contains subregions which contribute to conflict monitoring ${ }^{15,16,19,77,78}$. For instance, in a motion-based Simon task, in which the task-irrelevant motion direction of the target was either consistent or inconsistent with the response hand, the right PosCG was significantly activated ${ }^{19}$. Since the task-irrelevant motion direction in that study was defined by the relative positions between the moving dots, i.e., allocentric information, enhanced neural activity in the right PosCG in the incongruent condition (compared to the congruent condition) indicates the functional role of the right PosCG in resolving conflict specifically caused by irrelevant allocentric representations.

Specific mechanisms underlying the single-source Simon effect. The neural interaction contrast "EgoC $($ AlloIC $>$ AlloC) $>$ EgoIC (AlloIC $>$ AlloC)" revealed significant activations in a dorsolateral frontoparietal network, including the right posterior SPG (BA7) extending into the right SMA, the right SFG, and the right IFG (Fig. 2B and Table 1B). This right lateralized dorsolateral frontoparietal network was specifically involved in the single-source Simon effect, as indicated by increased neural activity when one type of spatial representation was incongruent while the other type was congruent with the response hand (i.e., in the EgoC_AlloIC and EgoIC_AlloC conditions), compared to when both spatial representations were congruent (EgoC_ AlloC) or both incongruent (EgoIC_ AlloIC) with the response hand (Fig. 2B).

Neural activations in the frontoparietal network have been shown to increase significantly during conflict processing $^{79-81}$. For example, significant effects of interference were found in the frontoparietal network, and their activation probably reflects response conflicts, cognitive control, and conflict detection ${ }^{82-86}$. Among the frontoparietal network, the right posterior SPG is critical for spatial location representations ${ }^{63,87-91}$ and the integration of spatial information from different reference frames during the localization of objects ${ }^{92-96}$. The right SPG also plays an essential role in manipulating spatial stimulus-response compatibility ${ }^{33,97-99}$. Besides, the right frontal brain regions have been suggested to be involved in the maintenance of spatial information during the processing of spatial relations ${ }^{100}$. Therefore, consistent with previous evidence, increased neural activity in the right frontoparietal network during the single-source Simon effect of the present study might be related to monitoring spatial information from multiple coordinate systems. Specifically speaking, in the current study, the allocentric and egocentric location of the target could either match (i.e., on the same side in the EgoC_ AlloC and the EgoIC_AlloIC condition) or mismatch (i.e., on different sides in the EgoC_AlloIC and EgoIC_AlloC condition) (Fig. 1A). Based on the observed pattern of neural activity in the right SPG, which is the maximum activation in the frontoparietal network, we found significantly higher neural activity in the two "Mismatch" conditions (EgoC_AlloIC and EgoIC_AlloC) than the two "Match" conditions (EgoC_AlloC and EgoIC_AlloIC) (Fig. 2B). According to our hypothesis, upon integrating spatial information from egocentric and allocentric reference coordinate systems into the common abstract spatial coordinate system, the right dorsolateral frontoparietal network might function as a mismatch detector between various sources of spatial information. This mismatch detector will be activated and generate mismatch signals whenever the allocentric and egocentric positions of the same object are on opposite sides.

Moreover, based on the results of the PPI analysis, the right SPG (the maximum neural activation in the frontoparietal network) showed enhanced functional interactions with the left ventral MT and the left ventral lateral IFG in the "Match" conditions, compared to the "Mismatch" conditions (Fig. 2C). Visual processing in the human brain is anatomically organized along two distinct streams: a dorsal stream, which is originating from the striate cortex and projecting to the posterior parietal gyrus, and a ventral stream, which is originating from the striate cortex and reaching the inferotemporal cortex ${ }^{67,101-103}$. Besides, these two visual streams are not only anatomically but also functionally distinct. External visual inputs are transformed into perceptual representations in the ventral visual stream, which enables the recognition of objects as well as their spatial relations, while visual information was transformed into the sensorimotor representations in the dorsal visual stream, which supports the planning and online control of visually guided actions in the environment ${ }^{1,67,102,104}$. Further anatomical studies showed that visual projections in the dorsal stream are extended from posterior parietal gyrus finally into the principal sulcus (BA 46) of the dorsolateral prefrontal cortex ${ }^{105-108}$, whereas projections in the ventral stream is extended from inferotemporal gyrus ultimately into the inferior convexity (BA 12 and 45) of the ventrolateral prefrontal corte ${ }^{109-111}$. Functionally, face processing activates the right IFC in the ventral stream, whereas location processing activates the superior frontal region in the ventral stream ${ }^{112}$. Indeed, the ventral stream projections to the MT and the IFC are exquisitely prepared to serve as an interface between vision and cognition ${ }^{113}$. Therefore, in the current study, the enhanced neural coupling between the right SPG in the dorsal and the ventral visual stream in the 
"Match" conditions may contribute to representing a common abstract spatial representation where egocentric and allocentric reference frame information converges, i.e., activating a general concept of left and right.

Please also note, the current neural activations in the dorsolateral frontoparietal network are mostly right lateralized (Fig. 2B and Table 1B). Numerous studies on healthy subjects and neurosurgical, brain-injured patients consistently reported that the right hemisphere was specialized in the visuospatial analysis of the external world $^{114-117}$. For example, unilateral inattention and spatial neglect occur much more frequently after right than left hemisphere (parietal, frontal, thalamic, basal ganglia) damage, in particular for lesions located in the right temporal-parietal and occipital junction ${ }^{118-120}$. Moreover, the right hemisphere has been found to be superior in discriminating the directional orientation of the body as well as body-part positional relationships ${ }^{115,117,121,122}$. In most of the previously reported studies on visuospatial processing, the spatial representations involved were based on one specific spatial coordinate system. In the current study, we further revealed that the right dorsolateral frontoparietal network monitors spatial representations not only in a specific spatial coordinate system but also in the high-level abstract coordinate map.

\section{Conclusion}

To summarize, by orthogonally combining the Simon effect based on allocentric and egocentric reference frames, we revealed general and specific neural correlates underlying egocentric and allocentric Simon conflicts. We found that the right PreCG, extending to the right SMA, is generally involved in the Simon conflict between spatial information and response code, irrespective of the spatial reference frame involved. Furthermore, right PosCG is specifically involved in the Simon conflict induced by task-irrelevant allocentric representations. Finally, our results suggest that the right dorsolateral frontoparietal network, including the right posterior SPG extending into the right IFG, may function as a mismatch detector to monitor the congruency between the two sources of spatial information with regard to the allocentric and egocentric reference frames.

\section{References}

1. Goodale, M. A., Milner, A. D. \& Melvyn, A. Separate visual pathways for perception and action. Trends in Neurosciences 15, 20-25 (1992).

2. Milner, A. D. \& Goodale, M. A. The Visual Brain in Action. Oxford; New York: Oxford University Press (1995).

3. Andersen, R. A. \& Buneo, C. A. Intentional Maps in Posterior Parietal Cortex. Annual Review of Neuroscience 25, 189-220 (2002).

4. Cohen, Y. E. \& Andersen, R. A. A common reference frame for movement plans in the posterior parietal cortex. Nature Reviews Neuroscience 3, 553-562 (2002).

5. Andersen, R. A. Multimodal integration for the representation of space in the posterior parietal cortex. Philosophical Transactions of the Royal Society B: Biological Sciences 352, 1421-1428 (1997).

6. Rolls, E. T. \& Xiang, J. Spatial view cells in the primate hippocampus and memory recall. 200, 1-26 (2006).

7. Simon, J. R. Reactions toward the source of stimulation. Journal of Experimental Psychology 81, 174-176 (1969).

8. Wühr, P. \& Ansorge, U. A Simon effect in memory retrieval: evidence for the response-discrimination account. Psychonomic bulletin \& review 14, 984-988 (2007).

9. Spironelli, C., Tagliabue, M. \& Umiltà, C. Response selection and attention orienting a computational model of Simon effect asymmetries. Experimental Psychology 56, 274-282 (2009).

10. Wang, H. et al. The Simon effect based on the egocentric and allocentric reference frame. Attention, Perception \& Psychophysics 78, 427-436 (2016).

11. Roswarski, T. E. \& Proctor, R. W. The role of instructions, practice, and stimulus-hand correspondence on the Simon effect. Psychological research 67, 43-55 (2003).

12. Wiegand, K. \& Wascher, E. Dynamic Aspects of Stimulus-Response Correspondence: Evidence for Two Mechanisms Involved in the Simon Effect. Journal of Experimental Psychology: Human Perception and Performance 31, 453-464 (2005).

13. Wallace, R. J. S-R compatibility and the idea of a response code. Journal of experimental psychology 88, 354-360 (1971).

14. Wühr, P. The Simon effect in vocal responses. Acta Psychologica 121, 210-226 (2006).

15. Peterson, B. S. et al. An event-related functional MRI study comparing interference effects in the Simon and Stroop tasks. 13, 427-440 (2002).

16. Fan, J., Flombaum, J. I., McCandliss, B. D., Thomas, K. M. \& Posner, M. I. Cognitive and Brain Consequences of Conflict. NeuroImage 18, 42-57 (2003).

17. Liu, X., Banich, M. T., Jacobson, B. L. \& Tanabe, J. L. Common and distinct neural substrates of attentional control in an integrated Simon and spatial Stroop task as assessed by event-related fMRI. NeuroImage 22, 1097-1106 (2004).

18. Nichols, T., Brett, M., Andersson, J., Wager, T. \& Poline, J. B. Valid conjunction inference with the minimum statistic. NeuroImage 25, 653-660 (2005).

19. Wittfoth, M., Buck, D., Fahle, M. \& Herrmann, M. Comparison of two Simon tasks: Neuronal correlates of conflict resolution based on coherent motion perception. NeuroImage 32, 921-929 (2006).

20. Nee, D. E., Wager, T. D. \& Jonides, J. Interference resolution: Insights from a meta-analysis of neuroimaging tasks. Cognitive, Affective \& Behavioral Neuroscience 7, 1-17 (2007).

21. Pomianowska, I., Germeys, F., Verfaillie, K. \& Newell, F. N. The role of social cues in the deployment of spatial attention: head-body relationships automatically activate directional spatial codes in a Simon task. Frontiers in Integrative Neuroscience 6, 1-7 (2012).

22. Avillac, M., Ben Hamed, S. \& Duhamel, J.-R. Multisensory Integration in the Ventral Intraparietal Area of the Macaque Monkey. Journal of Neuroscience 27, 1922-1932 (2007).

23. Hauser, P. C., Cohen, J., Dye, M. W. G. \& Bavelier, D. Visual constructive and visual - Motor skills in deaf native signers. Journal of Deaf Studies and Deaf Education 12, 148-157 (2007).

24. Rowland, B. A., Stanford, T. R. \& Stein, B. E. A model of the neural mechanisms underlying multisensory integration in the superior colliculus. Perception 36, 1431-1443 (2007).

25. Tversky, B. \& Hard, B. M. Embodied and disembodied cognition: Spatial perspective-taking. Cognition, https://doi.org/10.1016/j. cognition.2008.10.008 (2009).

26. Findlay, J. M., Brown, V. \& Gilchrist, I. D. Saccade target selection in visual search: The effect of information from the previous fixation. Vision Research 41, 87-95 (2001).

27. Friston, K. J., Penny, W. D. \& Glaser, D. E. Conjunction revisited. NeuroImage 25, 661-667 (2005).

28. Poline, J. B., Worsley, K. J., Evans, A. C. \& Friston, K. J. Combining spatial extent and peak intensity to test for activations in functional imaging. NeuroImage 5, 83-96 (1997).

29. Kriegeskorte, N., Simmons, W. K., Bellgowan, P. S. F. \& Baker, C. I. Circular analysis in systems neuroscience: the dangers of double dipping. Nat Neurosci 12, 535-540 (2009). 
30. Kriegeskorte, N., Simmons, W. K., Bellgowan, P. S. \& Baker, C. I. Circular inference in neuroscience: The dangers of double dipping. Journal of Vision 8, 88-88 (2010).

31. Crammond, D. J. \& Kalaska, J. F. Modulation of preparatory neuronal activity in dorsal premotor cortex due to stimulus-response compatibility. J Neurophysiol 71, 1281-1284 (1994).

32. Dassonville, P. et al. The Effect of Stimulus-Response Compatibility on Cortical Motor Activation. NeuroImage 13, 1-14 (2001).

33. Matsumoto, E., Misaki, M. \& Miyauchi, S. Neural mechanisms of spatial stimulus-response compatibility: The effect of crossedhand position. Experimental Brain Research 158, 9-17 (2004).

34. Koski, L., Molnar-Szakacs, I. \& Iacoboni, M. Exploring the contributions of premotor and parietal cortex to spatial compatibility using image-guided TMS. NeuroImage 24, 296-305 (2005).

35. Brass, M., Wenke, D., Spengler, S. \& Waszak, F. Neural Correlates of Overcoming Interference from Instructed and Implemented Stimulus-Response Associations. Journal of Neuroscience 29, 1766-1772 (2009).

36. Iacoboni, M., Woods, R. P., Lenzi, G. L. \& Mazziotta, J. C. Merging of oculomotor and somatomotor space coding in the human right precentral gyrus. Brain 120, 1635-1645 (1997).

37. Sylvester, C. Y. C. et al. Switching attention and resolving interference: fMRI measures of executive functions. Neuropsychologia 41, 357-370 (2003).

38. Mückschel, M., Stock, A., Dippel, G., Chmielewski, W. \& Beste, C. Interacting sources of interference during sensorimotor integration processes. NeuroImage, https://doi.org/10.1016/j.neuroimage.2015.09.075 (2015).

39. Cieslik, E. C., Mueller, V. I., Eickhoff, C. R., Langner, R. \& Eickhoff, S. B. Three key regions for supervisory attentional control: Evidence from neuroimaging meta-analyses. Neuroscience and Biobehavioral Reviews, https://doi.org/10.1016/j.neubiorev.2014.11.003 (2015).

40. Ridderinkhof, K. R. Micro- and macro-adjustments of task set: Activation and suppression in conflict tasks. Psychological Research https://doi.org/10.1007/s00426-002-0104-7 (2002).

41. Riehle, A. Spike Synchronization and Rate Modulation Differentially Involved in Motor Cortical Function. Science 278, 1950-1953 (1997).

42. Schluter, N. D., Rushworth, M. F. S., Passingham, R. E. \& Mills, K. R. Temporary interference in human lateral premotor cortex suggests dominance for the selection of movements. A study using transcranial magnetic stimulation. Brain 121, 785-799 (1998).

43. Murray, E. A., Bussey, T. J. \& Wise, S. P. Role of prefrontal cortex in a network for arbitrary visuomotor mapping. Experimental Brain Research 133, 114-129 (2000).

44. Simon, S. R. S. et al. Spatial attention and memory versus motor preparation: premotor cortex involvement as revealed by fMRI. Journal of neurophysiology 88, 2047-2057 (2002).

45. Ugur, H. C. et al. Arterial vascularization of primary motor cortex (precentral gyrus). Surgical Neurology 64, 48-52 (2005).

46. Stürmer, B., Leuthold, H., Soetens, E., Schröter, H. \& Sommer, W. Control over location-based response activation in the Simon task: Behavioral and electrophysiological evidence. Journal of Experimental Psychology: Human Perception and Performance, https://doi.org/10.1037//0096-1523.28.6.1345 (2002).

47. Stürmer, B. \& Leuthold, H. Control over response priming in visuomotor processing: A lateralized event-related potential study. Experimental Brain Research 153, 35-44 (2003).

48. Nieuwenhuis, S. \& Yeung, N. Neural mechanisms of attention and control: losing our inhibitions? Nature Neuroscience 8, $1631-1633$ (2005).

49. Egner, T., Delano, M. \& Hirsch, J. Separate conflict-specific cognitive control mechanisms in the human brain. NeuroImage 35, 940-948 (2007).

50. Verleger, R., Kuniecki, M., Möller, F., Fritzmannova, M. \& Siebner, H. R. On how the motor cortices resolve an inter-hemispheric response conflict: An event-related EEG potential-guided TMS study of the flankers task. European Journal of Neuroscience 30, 318-326 (2009).

51. Chmielewski, W. X., Mückschel, M., Roessner, V. \& Beste, C. Expectancy effects during response selection modulate attentional selection and inhibitory control networks. Behavioural Brain Research 274, 53-61 (2014).

52. Klein, P. A., Petitjean, C., Olivier, E. \& Duque, J. Top-down suppression of incompatible motor activations during response selection under conflict. NeuroImage 86, 138-149 (2014).

53. Stock, A. K., Wascher, E. \& Beste, C. Differential Effects of Motor Efference Copies and Proprioceptive Information on Response Evaluation Processes. PLoS ONE, https://doi.org/10.1371/journal.pone.0062335 (2013).

54. Mostofsky, S. H. \& Simmonds, D. J. Response inhibition and response selection: Two sides of the same coin. Journal of Cognitive Neuroscience, https://doi.org/10.1162/jocn.2008.20500 (2008).

55. Nachev, P., Kennard, C. \& Husain, M. Functional role of the supplementary and pre-supplementary motor areas. Nature Reviews Neuroscience 9, 856-869 (2008).

56. Kornblum, S., Hasbroucq, T. \& Osman, A. Dimensional overlap: cognitive basis for stimulus-response compatibility-a model and taxonomy. Psychological review 97, 253-270 (1990).

57. Kornblum, S. The way irrelevant dimensions are processed depends on what they overlap with: The case of Stroop-and Simon-like stimuli. Psychological Research 56, 130-135 (1994).

58. Neggers, S. F. W., Van der Lubbe, R. H. J., Ramsey, N. F. \& Postma, A. Interactions between ego- and allocentric neuronal representations of space. NeuroImage 31, 320-331 (2006).

59. Zhang, M. et al. Interaction between allocentric and egocentric reference frames in deaf and hearing populations. Neuropsychologia 54, 68-76 (2014).

60. Liu, N., Li, H., Su, W. \& Chen, Q. Common and specific neural correlates underlying the spatial congruency effect induced by the egocentric and allocentric reference frame. Human Brain Mapping 38, 2112-2127 (2017).

61. Grefkes, C., Weiss, P. H., Zilles, K. \& Fink, G. R. Crossmodal processing of object features in human anterior intraparietal cortex: An fMRI study implies equivalencies between humans and monkeys. Neuron 35, 173-184 (2002).

62. Gottlieb, J. From thought to action: the parietal cortex as a bridge between perception, action, and cognition. Neuron 53, 9-16 (2007).

63. Galati, G. et al. The neural basis of egocentric and allocentric coding of space in humans: a functional magnetic resonance study. Experimental Brain Research 133, 156-164 (2000).

64. James, T. W., Culham, J., Humphrey, G. K., Milner, A. D. \& Goodale, M. A. Ventral occipital lesions impair object recognition but not object-directed grasping: An fMRI study. Brain 126, 2463-2475 (2003).

65. Booth, J. R. et al. Neural development of selective attention and response inhibition. NeuroImage 20, 737-751 (2003).

66. Zaehle, T. et al. The neural basis of the egocentric and allocentric spatial frame of reference. Brain Research 1137, 92-103 (2007).

67. Milner, A. D. \& Goodale, M. A. Two visual systems re-viewed. Neuropsychologia 46, 774-785 (2008).

68. Medina, J. et al. Neural Substrates of Visuospatial Processing in Distinct Reference Frames: Evidence from Unilateral Spatial Neglect. Journal of Cognitive Neuroscience 21, 2073-2084 (2010).

69. Makuuchi, M., Someya, Y., Ogawa, S. \& Takayama, Y. Hand shape selection in pantomimed grasping: interaction between the dorsal and the ventral visual streams and convergence on the ventral premotor area. Human brain mapping 33, 1821-1833 (2012).

70. Cloutman, L. L. Interaction between dorsal and ventral processing streams: where, when and how? Brain and language 127, 251-263 (2013). 
71. Migliaccio, R. et al. Functional Connectivity of Ventral and Dorsal Visual Streams in Posterior Cortical Atrophy. Journal of Alzheimer's disease: JAD 51, 1119-1130 (2016).

72. Chen, Q., Weidner, R., Weiss, P. H., Marshall, J. C. \& Fink, G. R. Neural Interaction between Spatial Domain and Spatial Reference Frame in Parietal-Occipital Junction. Journal of Cognitive Neuroscience 24, 2223-2236 (2012).

73. Pause, M., Kunesch, E., Binkofski, F. \& Freund, H. J. Sensorimotor disturbances in patients with lesions of the parietal cortex. Brain 112, 1599-1625 (1989).

74. Binkofski, F. et al. Human anterior intraparietal area subserves prehension: A combined lesion and functional MRI activation study. Neurology 50, 1253-1259 (1998).

75. Binkofski, F., Kunesch, E., Classen, J., Seitz, R. J. \& Freund, H. J. Tactile apraxia: unimodal apractic disorder of tactile object exploration associated with parietal lobe lesions. 124, 132-44 (2001).

76. Binkofski, F. et al. Human anterior intraparietal area subserves prehension: A combined lesion and functional MRI activation study. Neurology, 50(5), 1253-1259 (1998).

77. Maclin, E. L., Gratton, G. \& Fabiani, M. Visual spatial localization conflict: an fMRI study. Neuroreport 12, 3633-3636 (2001).

78. Wang, P., Fuentes, L. J., Vivas, A. B. \& Chen, Q. Behavioral and neural interaction between spatial inhibition of return and the Simon effect. Frontiers in Human. Neuroscience 7, 572 (2013).

79. Schumacher, E. H., Elston, P. A. \& D’Esposito, M. Neural Evidence for Representation-Specific Response Selection. Journal of Cognitive Neuroscience 15, 1111-1121 (2003).

80. Cohen Kadosh, R., Cohen Kadosh, K., Henik, A. \& Linden, D. E. J. Processing conflicting information: Facilitation, interference, and functional connectivity. Neuropsychologia, https://doi.org/10.1016/j.neuropsychologia.2008.05.025 (2008).

81. Jin, Y., Olk, B. \& Hilgetag, C. C. Contributions of human parietal and frontal cortices to attentional control during conflict resolution: A 1-Hz offline rTMS study. Experimental Brain Research, https://doi.org/10.1007/s00221-010-2336-x (2010).

82. Kerns, J. G. Anterior Cingulate Conflict Monitoring and Adjustments in Control. Science 303, 1023-1026 (2004).

83. MacDonald, A. W., Cohen, J. D., Stenger, V. A. \& Carter, C. S. Dissociating the role of the dorsolateral prefrontal and anterior cingulate cortex in cognitive control. Science (New York, N.Y.) 288, 1835-1838 (2000).

84. Longworth, C. E. et al. NIH Public Access. Brain and Language 25, 1113-21 (2015)

85. Miller, E. K. \& Cohen, J. D. An Integrative Theory Of Prefrontal Cortex Function. 167-202 (2001).

86. Ridderinkhof, K. R., Ullsperger, M., Crone, E. A. \& Nieuwenhuis, S. The role of the medial frontal cortex in cognitive control. Science 306, 443-7 (2004).

87. Galati, G., Pelle, G., Berthoz, A. \& Committeri, G. Multiple reference frames used by the human brain for spatial perception and memory. Experimental Brain Research 206, 109-120 (2010).

88. Gross, C. G. \& Graziano, M. S. A. Review $\square$ : Multiple Representations of Space in the Brain. The Neuroscientist 1, 43-50 (1995).

89. Vallar, G. et al. A fronto-parietal system for computing the egocentric spatial frame of reference in humans. Experimental Brain Research 124, 281-286 (1999).

90. Committeri, G. et al. Reference Frames for Spatial Cognition: Different Brain Areas are Involved in Viewer-, Object-, and Landmark-Centered Judgments About Object Location. Journal of Cognitive Neuroscience 16, 1517-1535 (2004).

91. Buneo, C. A. \& Andersen, R. A. The posterior parietal cortex: Sensorimotor interface for the planning and online control of visually guided movements. Neuropsychologia 44, 2594-2606 (2006).

92. Andersen, R. A., Snyder, L. H., Bradley, D. C. \& Xing, J. Multimodal Representation of Space in the Posterior Parietal Cortex and Its Use in Planning Movements. Annual Review of Neuroscience 20, 303-330 (1997).

93. Shafritz, K. M., Gore, J. C. \& Marois, R. The role of the parietal cortex in visual feature binding. Proceedings of the National Academy of Sciences of the United States of America 99, 10917-10922 (2002).

94. Walter, E. \& Dassonville, P. Visuospatial contextual processing in the parietal cortex: An fMRI investigation of the induced Roelofs effect. NeuroImage 42, 1686-1697 (2008).

95. Wilber, A. A., Clark, B. J., Forster, T. C., Tatsuno, M. \& Mcnaughton, B. L. Interaction of Egocentric and World-Centered Reference Frames in the Rat Posterior Parietal. Cortex. 34, 5431-5446 (2014).

96. Calton, J. L. \& Taube, J. S. Where am I and how will I get there from here? A role for posterior parietal cortex in the integration of spatial information and route planning. 91, 186-196 (2010).

97. Iacoboni, M., Woods, R. P. \& Mazziotta, J. C. Brain-behavior relationships: evidence from practice effects in spatial stimulusresponse compatibility. Journal of neurophysiology 76, 321-31 (1996).

98. Merriam, E. P. et al. Stimulus-Response Incompatibility Activates Cortex Proximate to Three Eye Fields. NeuroImage 13, 794-800 (2001).

99. Schumacher, E. H. \& D'Esposito, M. Neural implementation of response selection in humans as revealed by localized effects of stimulus-response compatibility on brain activation. Human Brain Mapping 17, 193-201 (2002).

100. Han, J., Cao, B., Cao, Y. \& Gao, H. The role of right frontal brain regions in integration of spatial relation. Neuropsychologia 86, $29-37$ (2016)

101. Macko, K. A. et al. Mapping the primate visual system with [2-14C]deoxyglucose. Science (New York, N. Y.) 218, 394-397 (1982).

102. Mishkin, M., Ungerleider, L. G. \& Kathleen, A. Object vision and spatial vision: two cortical p hways. Trends in Neurosciences 6 , 414-417 (1983).

103. Haxby, J. V. et al. Dissociation of object and spatial visual processing pathways in human extrastriate cortex. Proceedings of the National Academy of Sciences 88, 1621-1625 (1991).

104. Goodale, M. A. \& Westwood, D. A. An evolving view of duplex vision: Separate but interacting cortical pathways for perception and action. Current Opinion in Neurobiology 14, 203-211 (2004).

105. Barbas, H. \& Mesulam, M. M. Cortical afferent input to the principalis region of the rhesus monkey. Neuroscience 15, 619-637 (1985).

106. Cavada, C. \& Goldman-Rakic, P. S. Posterior parietal cortex in rhesus monkey: I. Parcellation of areas based on distinctive limbic and sensory corticocortical connections. Journal of Comparative Neurology 287, 393-421 (1989).

107. Cavada, C. \& Goldman-Rakic, P. S. Posterior parietal cortex in rhesus monkey: II. Evidence for segregated corticocortical networks linking sensory and limbic areas with the frontal lobe. Journal of Comparative Neurology 287, 422-445 (1989).

108. Ungerleider, L. G. \& Desimone, R. Projections to the superior temporal sulcus from the central and peripheral field representations of V1 and V2. Journal of Comparative Neurology 248, 147-163 (1986).

109. Chavis, D. A. \& Pandya, D. N. Further observations on corticofrontal connections in the rhesus monkey. Brain Research 117, 369-386 (1976).

110. Ungerleider, L. G., Gaffan, D. \& Pelak, V. S. Projections from inferior temporal cortex to prefrontal cortex via the uncinate fascicle in rhesus monkeys. Experimental Brain Research 76, 473-484 (1989).

111. Webster, M. J., Bachevalier, J. \& Ungerleider, L. G. Connections of inferior temporal areas TEO and TE with parietal and frontal cortex in macaque monkeys. Cerebral Cortex 4, 470-483 (1994).

112. Ungerleider, L. G., Courtney, S. M. \& Haxby, J. V. A neural system for human visual working memory. Proceedings of the National Academy of Sciences of the United States of America 95, 883-90 (1998).

113. Lee, J. H. \& Van Donkelaar, P. Dorsal and ventral visual stream contributions to perception-action interactions during pointing. Experimental Brain Research 143, 440-446 (2002).

114. Benton, A. \& Tranel, D. Visuoperceptual, visuospatial, and visuoconstructive disorders. In Clinical neuropsychology (1993). 
115. Joseph, R. The right cerebral hemisphere: Emotion, music, visual-spatial skills, body-image, dreams, and awareness. Journal of Clinical Psychology, doi:10.1002/1097-4679(198809)44:5<630::AID-JCLP2270440502>3.0.CO;2-V (1988).

116. Foxe, J. J., McCourt, M. E. \& Javitt, D. C. Right hemisphere control of visuospatial attention: Line-bisection judgments evaluated with high-density electrical mapping and source analysis. NeuroImage, https://doi.org/10.1016/S1053-8119(03)00057-0 (2003).

117. Shulman, G. L. et al. Right Hemisphere Dominance during Spatial Selective Attention and Target Detection Occurs Outside the Dorsal Frontoparietal Network. Journal of Neuroscience, https://doi.org/10.1523/jneurosci.4085-09.2010 (2010).

118. Bisiach, E., Capitani, E. \& Porta, E. Two basic properties of space representation in the brain: evidence from unilateral neglect. Journal of Neurology, Neurosurgery \& Psychiatry 48, 141-144 (1985).

119. Vallar, G. \& Perani, D. The anatomy of unilateral neglect after right-hemisphere stroke lesions. A clinical/CT-scan correlation study in man. Neuropsychologia, https://doi.org/10.1016/0028-3932(86)90001-1 (1986).

120. Laeng, B. Constructional apraxia after left or right unilateral stroke. Neuropsychologia. https://doi.org/10.1016/j. neuropsychologia.2006.01.023 (2006).

121. Nebes, R. D. \& Sperry, R. W. Hemispheric deconnection syndrome with cerebral birth injury in the dominant arm area. Neuropsychologia, https://doi.org/10.1016/0028-3932(71)90020-0 (1971).

122. Sperry, R. Some effects of disconnecting the cerebral hemispheres. Science, https://doi.org/10.1126/science.7112125 (1982).

\section{Acknowledgements}

The work was supported by grants from Natural Science Foundation of China (31871138, 31700975). QC is supported by the Program for New Century Excellent Talents in the University of China (NCET-12-0645) and by the Chang Jiang Scholars Program (2016).

\section{Author Contributions}

Ralph Weidner, Gereon R. Fink, and Qi Chen designed the experiments and edited the manuscript; Hui Li, Nan Liu and You Li conducted the experiments; Hui Li processed the data and drafted the article.

\section{Additional Information}

Competing Interests: The authors declare no competing interests.

Publisher's note Springer Nature remains neutral with regard to jurisdictional claims in published maps and institutional affiliations.

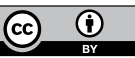

Open Access This article is licensed under a Creative Commons Attribution 4.0 International License, which permits use, sharing, adaptation, distribution and reproduction in any medium or format, as long as you give appropriate credit to the original author(s) and the source, provide a link to the Creative Commons license, and indicate if changes were made. The images or other third party material in this article are included in the article's Creative Commons license, unless indicated otherwise in a credit line to the material. If material is not included in the article's Creative Commons license and your intended use is not permitted by statutory regulation or exceeds the permitted use, you will need to obtain permission directly from the copyright holder. To view a copy of this license, visit http://creativecommons.org/licenses/by/4.0/.

(C) The Author(s) 2019 